\title{
Appropriate Smart Factory for SMEs: Concept, Application and Perspective
}

\author{
Woo-Kyun Jung ${ }^{1} \cdot$ Dong-Ryul Kim ${ }^{2} \cdot$ Hyunsu Lee ${ }^{2} \cdot$ Tae-Hun Lee $^{3} \cdot$ Insoon Yang ${ }^{4} \cdot$ Byeng D. Youn $^{2,5} \cdot$ Daniel Zontar $^{3}$. \\ Matthias Brockmann ${ }^{3} \cdot$ Christian Brecher ${ }^{3} \cdot$ Sung-Hoon $\mathrm{Ahn}^{2,5}$
}

Received: 26 October 2020 / Revised: 15 November 2020 / Accepted: 17 November 2020 / Published online: 8 December 2020

(c) The Author(s) 2020

\begin{abstract}
In the manufacturing industry, the smart factory is considered the final stage of the Fourth Industrial Revolution. Manufacturing companies are pursuing breakthroughs by introducing various advanced technologies to ensure their competitiveness. However, it is difficult for small and medium-sized enterprises (SMEs) to adopt smart-factory technologies, owing to financial and technical burdens. This paper proposes a smart factory that can be applied technically and strategically to the introduction of a smart factory for SMEs. The concept of an 'appropriate smart factory' involves applying appropriate measures in terms of cost and scale with consideration of the situations faced by SMEs. The goal is to build a smart factory that has necessary functions (Essential) but can be easily operated (Simple) at a low cost (Affordable) and has compatibility (Interoperable). This paper presents technical application measures such as appropriate smart sensors, appropriate IoT (Internet of Things), and small data processing, along with the definition of an appropriate smart factory. In addition, a case study was examined where the quality inspection equipment for garment manufacturing SMEs was developed by applying the appropriate smart factory concept.
\end{abstract}

Keywords Appropriate smart factory $\cdot$ Small and medium-sized enterprises $\cdot$ Interoperable $\cdot$ Sensor $\cdot$ Edge computing . Artificial intelligence $\cdot$ Internet of Things

\section{Introduction}

In the era of the Fourth Industrial Revolution, global manufacturers are striving to gain competitive advantages by applying cutting-edge technologies such as the Internet of Things (IoT), robotics, artificial intelligence (AI), Big Data, and cyber-physical systems [1, 2]. In particular,

Sung-Hoon Ahn

ahnsh@snu.ac.kr

1 Soft Robotics Research Center, Seoul National University, Seoul, Republic of Korea

2 Department of Mechanical Engineering, Seoul National University, Room 1405, Building 301, 1 Gwanak-ro, Gwanak-gu, Seoul 08826, Republic of Korea

3 Fraunhofer Institute for Production Technology IPT, Aachen, Germany

4 Department of Electrical and Computer Engineering, Seoul National University, Seoul, Republic of Korea

5 Institute of Advanced Machines and Design, Seoul National University, Seoul, Republic of Korea large enterprises implement autonomous, networked smart factories aimed at improving productivity and quality for maintaining their dominance in the global market [3]. In an advanced smart factory, all manufacturing resources are monitored in real time over the Internet, and production activities can be controlled autonomously [4]. A smart factory is developed to remotely diagnose the conditions of production sites and predict future conditions, as well as to engage in the entire plant operation process-from product design and production to logistics and disposal [5, 6].

Meanwhile, trends such as energy reduction and ecofriendly processing, which have recently emerged as global issues, are also becoming important smart-factory topics. Along with research on the energy efficiency in the manufacturing process [7-10], various studies on eco-friendly factors of manufacturing and products and sensor development for environmental evaluation have been conducted [11-14]. However, most of the research on energy and the environment is concerned with measuring the efficiency of energy consumption or environmental pollution. 
Small and medium-sized enterprises (SMEs) face many difficulties in realising such smart factories. For the case of manufacturing enterprises in Korea, SMEs are defined as companies with total assets of $<440$ million USD, and $97.9 \%$ of the manufacturers in Korea are SMEs [15]. Although some SMEs have strong global competitiveness based on advanced technology, most of SMEs have weaker business-oriented financial structures and technical capabilities than large companies. Such financial and technical constraints hinder the adoption of smart factories by the SMEs. Figure 1 shows the survey results for the difficulties of smart-factory promotion among Korean companies [16]. While SMEs have cited financial burdens and lack of technology as the most significant obstacles for adopting smart factories, large enterprises are more concerned with the lack of Big Data. Additionally, SMEs require an adoption strategy that is consistent with their circumstances and the purpose of introducing smart factories [17].

This paper proposes the concept of an 'appropriate smart factory,' which can be applied with small financial and technical burdens for SMEs to promote smart factories. We establish the concept of an appropriate smart factory by considering the key issues of SMEs and present measures that can be implemented in various technologies, such as sensors, IoT, process, data-processing system, and robotics. In the application of smart factories, attempts to reflect the needs of SMEs have been continuously made [18]. This paper provides useful guidance to many SMEs by presenting concepts and methods for appropriate smart factories that can be practically referenced by SMEs for the adoption of smart-factory technologies. For most SMEs, except for some that have strong competitiveness based on advanced technology, the issues covered in this paper can be useful references.

The remainder of this paper is organized as follows; Sect. 2 presents the latest research trends linked to the promotion of smart factories for SMEs, and Sect. 3 presents the concept of appropriate smart factories and technical implementation plans. Section 4 presents examples of the development of vision-based quality inspection equipment based on the requirements of small and medium-sized clothing manufacturing companies as case studies of implementing the appropriate smart factory concept. Section 5 discusses additional matters regarding the implementation of an appropriate smart factory, and Sect. 6 concludes the paper.

\section{Literature Review}

In 2010, Zuehlk introduced the SmartFactory ${ }^{\mathrm{KL}}$ Initiative as a proposal for new solutions for future factory technologies with their demonstration and research testbeds [19]. Since then, various efforts have been made to realise the future of manufacturing plants with advanced technologies under the theme of smart factories or smart manufacturing [20-23]. Kim et al. organised and presented the development direction of smart manufacturing trends involving machine learning for various types of manufacturing equipment [24], and Shin et al. proposed a diagnostic and soundness management framework applicable for smart factories [25]. Kim et al. proposed a real-time monitoring and diagnosis system based on AI and data that is applicable to manufacturing equipment [26]. Pham and Ahn investigated high-precision industrial robot parts applicable to smart factories [27]. However, the application of Fourth Industrial Revolution technology is still being pursued in a way that is suitable for large enterprises rather than SMEs [28] and still has many barriers to be applied to SMEs [29].

Recently, concerns have been raised over how to apply smart factories with consideration of the structures and resources of SMEs [30]. Mittal et al. presented the characteristics of SMEs and the research gaps that must be addressed to successfully support SMEs, e.g. roadmaps, maturity models, frameworks, and readiness assessments [31]. Issa et al. proposed technology matching among collaborative enterprises, financial and technical government support, project management system, etc. [32]. Chonsawat et al. presented manufacturing and operation strategies,
Fig. 1 Obstacles to promoting smart factory [16]

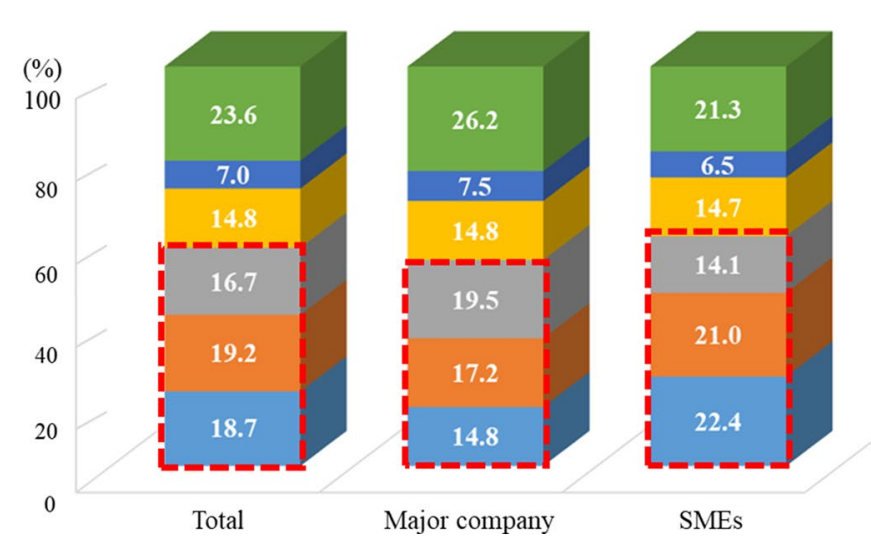

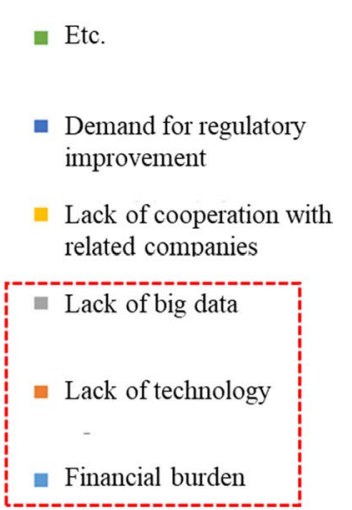


manpower capabilities, technology-driven processes, business and organisational strategies, etc. for promoting smart factories among SMEs [33].

Smart-factory technology has been applied to SMEs in various ways. Ghobakhlu et al. developed a model for SME adoption of smart factories through the integration of smart manufacturing information and digital technologies such as AI and Big Data with their business operations [34]. Jun et al. proposed a platform to support smart manufacturing through cloud applications [35]. Menez et al. developed a real-time manufacturing system through improvements to SMEs' Manufacturing Execution System for smart-factory application [36]. Kolla et al. presented a junction with the lean technique, a traditional manufacturing efficiency model, for SME smart-factory application [37].

Recent literature indicates that SMEs' efforts to introduce smart factories are mostly high-level frameworks or have considerable administrative access to internal and external elements. SMEs, whose structural and financial bases are more core business-oriented than those of large enterprises, must begin the adoption of a smart factory with the introduction of acceptable technologies that can be applied directly at their manufacturing sites.

\section{Concept of Appropriate Smart Factory for SMEs}

The concept of the appropriate smart factory involves applying a method suitable in terms of cost and scale to the smart factory to suit the situation in which the technology is used. It aims to build a smart factory that has essential functions but is easy to operate and low-cost. The appropriate smart factory elements can be defined as 'Essential, Affordable, Simple, and Interoperable' (EASI).

'Essential' refers to the composition of the technology to the level of essential functions of the equipment or processes that small and medium enterprises intend to adopt. Higherthan-necessary specifications can lead to heavy systems with high costs. For example, a high performance computer is not appropriate if factory operational information is generated in the form of simple numerical data. It is often possible to process data adequately using small computers (microcontroller units), e.g. Arduino or Raspberry Pi. Thus, it is desirable to adopt a tool with appropriate specifications depending on the circumstances.

'Affordable' implies that the introduction of a smart factory requires consideration of whether the company can accept the financial burden and whether the cost-effectiveness is appropriate. As mentioned previously, SMEs recognise that the financial sector is the largest burden. This is related to sustainability, which can face significant challenges if the introduction of technologies creates a lower return on investment (ROI).

'Simple' implies that the smart-factory technology or facilities should be easy to install, operate, and maintain. If excessive additional infrastructure must be added to the installation or if additional experts are needed owing to difficulties in operation and repair, a burden is placed on the SME. Thus, by introducing smart-factory technology with a simple system configuration, operation method, and repair method, the technology can be operated efficiently without additional personnel and job training.

Finally, 'Interoperability' refers to the utilization of facilities independent of their hardware, operation system, sensor type, data structure, and communication standard. The introduction of new facilities or technologies often necessitates separate facilities for integrated operations, unless compatibility with existing ones is considered. This can increase the production costs.

Table 1 presents the aforementioned appropriate smart factory elements and considerations. If these considerations are carefully reviewed and reflected in the introduction of smart-factory technology, SMEs can implement smart-factory solutions with a minimal burden.

Figure 2 shows the scope of the appropriate smart factory elements in the concept of a general smart factory. The smart factory collects data from all the facilities and environments of the factory through sensors and processes them at the site level or generates Big Data using IoT and sends the data to the cloud. Stored data analyses the phenomena of factories and makes decisions using AI. Subsequently, the physical world site is controlled autonomously in the factory. Appropriate smart factory elements can be applied to all parts of a smart factory, including the equipment and processes, sensors, IoT, and data processing. The sections below describe the application of the appropriate smart factory elements in further detail.

Table 1 Appropriate smart factory factors and considerations

\begin{tabular}{ll}
\hline Factors & Key considerations \\
\hline Essential & $\begin{array}{l}\text { Composed only of necessary technologies } \\
\text { Without over-specs compared with the product } \\
\text { produced } \\
\text { Adoptable given the company's finances } \\
\text { Adfordable }\end{array}$ \\
Simple & $\begin{array}{l}\text { Easy to install and maintain } \\
\text { Easily operated by the operator }\end{array}$ \\
Interoperable & $\begin{array}{l}\text { Data interchangeable between systems in the factory } \\
\text { Parts or components interchangeable in the factory }\end{array}$ \\
\hline
\end{tabular}


Fig. 2 Concept and characteristics of appropriate smart factory

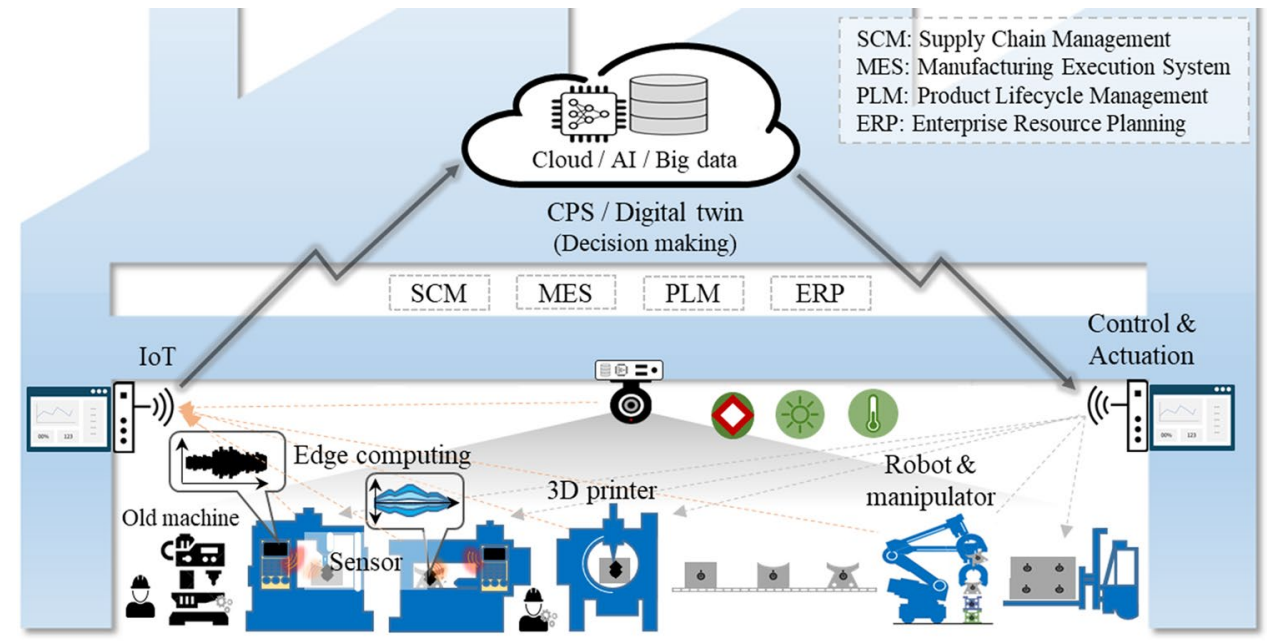

General smart factory

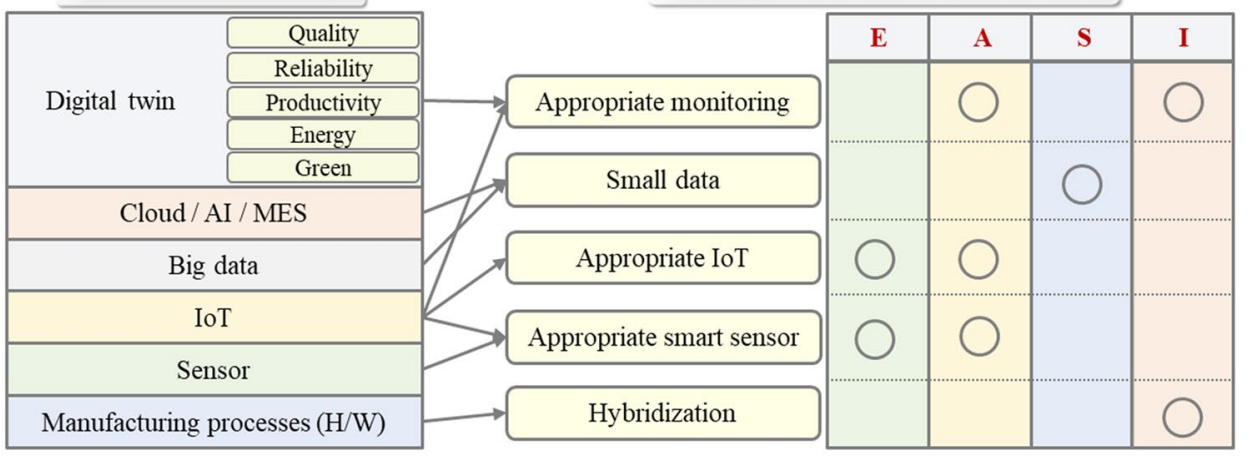

Essential Affordable Simple Interoperable

\subsection{Appropriate Smart Sensor}

The sensor that collects the production and status data of manufacturing facilities is the starting point of the smart factory and connects the factory to the data. Owing to the cost of installing and operating sensors and the additional equipment required, SMEs have concerns regarding the use of sensors to collect manufacturing data [38]. Sensors that are affordable and appropriate for collecting data are the most important requirements for SMEs to adopt smart factories.

The use of highly specified ultraprecision vibration sensors for measuring machine-tool vibrations allows precise sensitivity measurements, but such sensors are expensive. If the reason for measuring the vibration is not to ensure the high quality of the workpiece but to identify trends in the process and only an intermediate sensitivity level is needed to prevent the generation of defective products, it is not mandatory to purchase and install highly-specified vibration sensors. There are two options for resolving this issue: (a) control data and (b) a cheap sensor. For controlled systems such as an industrial robot, control data can be an option for an appropriate smart factory but exhibit low sensitivity. Another option is an appropriate smart sensor. Figure 3 presents an example of an appropriate smart sensor manufactured by printing nanoparticles directly into the parent metal. The multifunction sensor can simultaneously measure trends in both low and high levels of strain and vibration $[39,40]$. It can be produced at a cost of several dollars (in contrast to high-end vibration sensors, which have a production cost of thousands of dollars), and its sensitivity is sufficient for a simple analysis of the process quality. The development and dissemination of appropriate smart sensors can facilitate the adoption of smart factories by SMEs.

\subsection{Appropriate loT}

IoT devices are cloud-connected sensors and physical objects that collect data from manufacturing facilities and transmit the data to servers or processing them directly through edge computing. In some cases, IoT devices are configured with sensors directly included in existing facilities, and in others, they receive data from separate sensors and process or transmit the data. Commercial IoT equipment is expensive for SMEs to adopt, as it has various functions. The availability of various functions can be over-specified, adding to the weight of the system.

Low-cost computing devices, e.g. Arduino, with wireless communication modules are combined to produce 
Fig. 3 Direct-printed smart sensor: low-cost and multifunctional

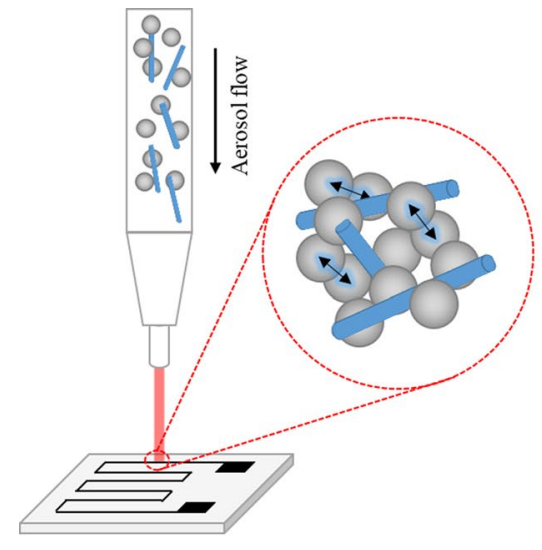

appropriate IoT devices. Figure 4 shows examples of measuring the consumed power of the equipment, e.g. machine tools, three-dimensional printers, sewing machines, and laser processing equipment. Using low-cost small computers, IoT devices can be manufactured with sufficient data collection and communication characteristics compared with expensive equipment at low cost. Additionally, the powerconsumption measurement results can be used to monitor the status of the connected equipment and check the output in real time [29].

\subsection{Small Data Processing}

In the smart-factory system, manufacturing data collected by sensors are stored in the cloud or local (on-premise) server. Data may not have a meaning until information is extracted through visualisation, data processing, or aggregation of multiple sources. Big Data and AI technologies are used

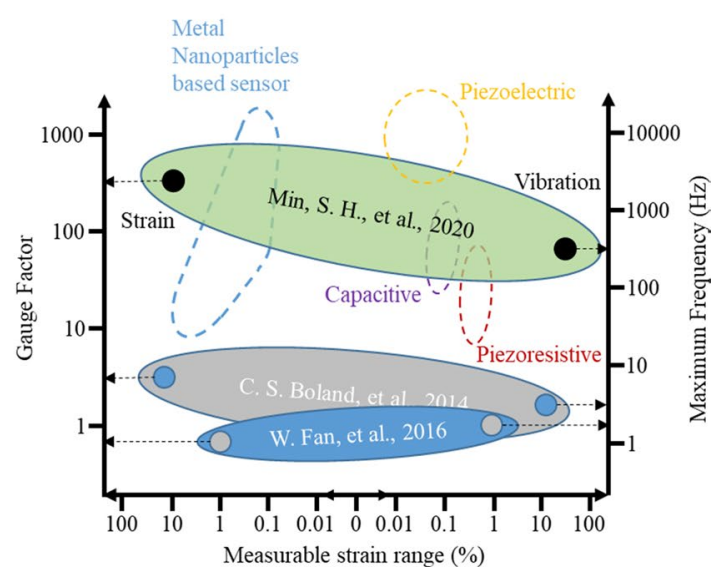

for processing these data. However, the cost of developing Big Data and AI technologies and the need for professional labour are significant obstacles for SMEs. The appropriate smart factory approach involves reducing costs by transposing data before the collection of Big Data. It is recommended to extract characteristic key values from the data via statistical techniques or physical-quantity calculations. These approaches often provide valuable insights, and tooling, interpretation, and implementation are often easier to realise than full AI models.

Figure 5 shows how garment manufacturers' sewing worksites can use statistical techniques to approximate power data profiles to pattern their work characteristics continuously according to time-series data [41]. Using this method, it was possible to analyse the amount and time of work per hour for the sewing worker. Table 2 compares the results of two AI techniques-the convolutional neural network $(\mathrm{CNN})$ and small data processing-for the same

Fig. 4 Appropriate IoT device for power consumption monitoring

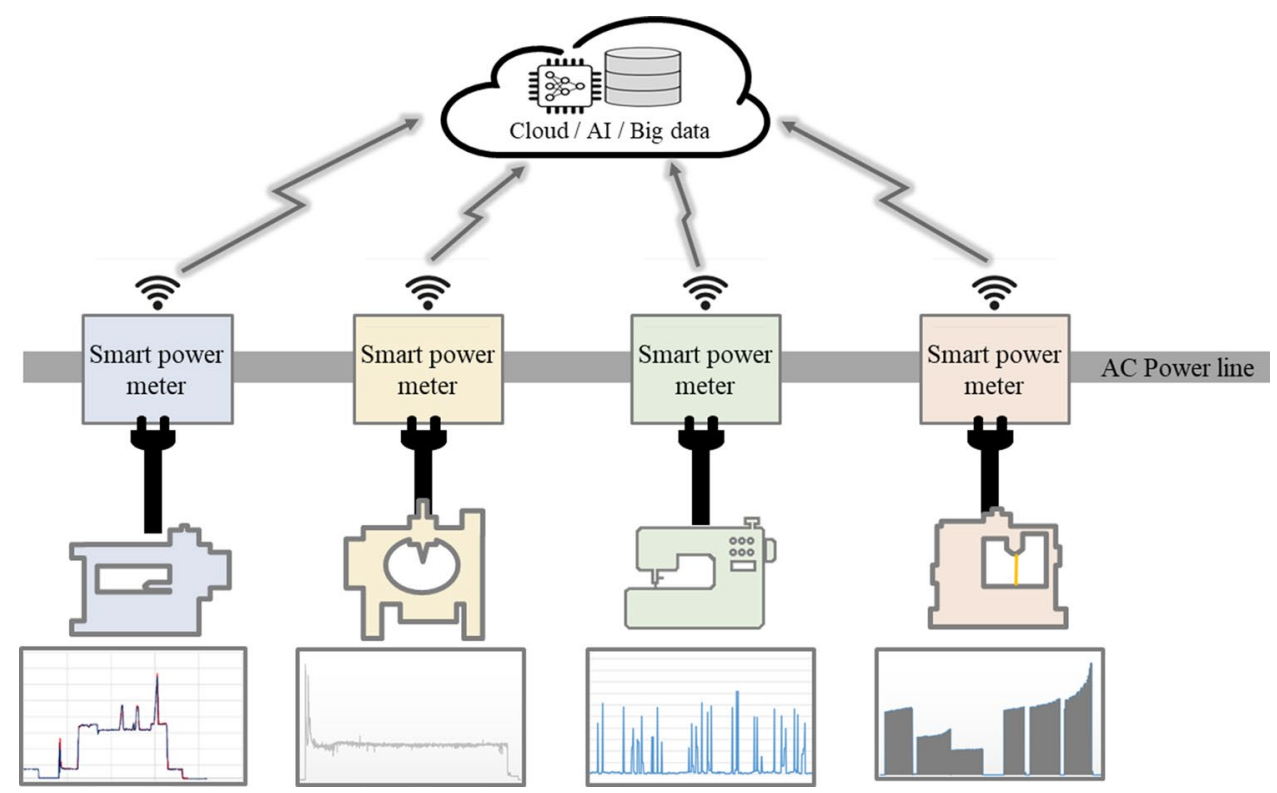


Fig. 5 Power consumption data analysis using small data processing
Table 2 Comparison between the AI-based method and small data processing

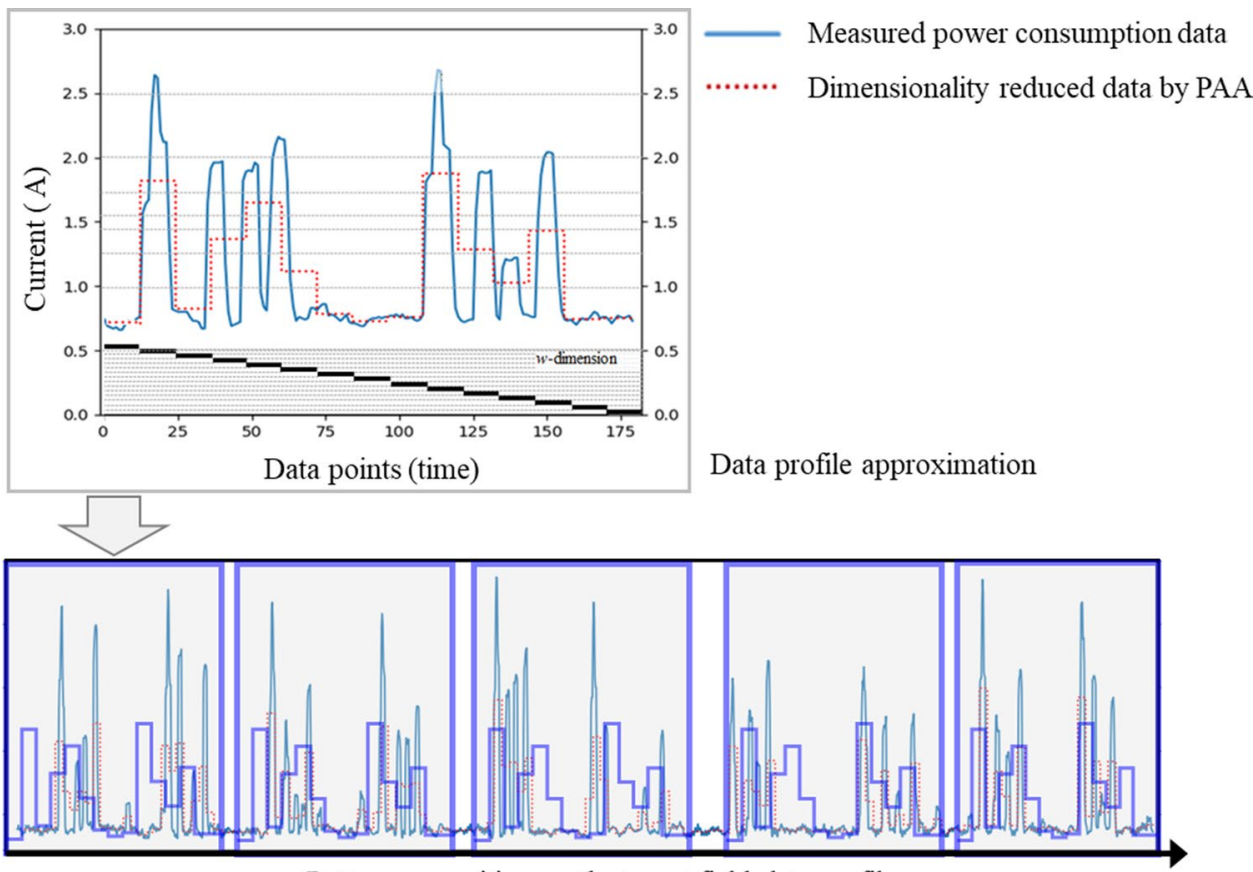

Pattern recognition on the target field data profile

\begin{tabular}{lll}
\hline Category & AI (CNN) & $\begin{array}{l}\text { Small data process- } \\
\text { ing (approximation } \\
\text { method) }\end{array}$ \\
\hline Data extraction/train (1 worker) & $\sim 90 \mathrm{~min}$ & $\sim 1 \mathrm{~min}$ \\
Calculation time (1 day of work for 1 worker) & $<20 \mathrm{~s}$ & $<3 \mathrm{~s}$ \\
Line level analysis time (1 production line) & $\sim 9$ days & $\sim 1 \mathrm{~h}$ \\
Pattern-recognition accuracy & $\sim 88 \%$ & $\sim 92 \%$ \\
\hline
\end{tabular}

power-consumption data for the sewing line. When data for one production line in which $>50$ workers worked simultaneously were analysed, CNN spent nearly 9 days, whereas the small data processing required approximately $1 \mathrm{~h}$. Additionally, the pattern-recognition accuracy was higher in the small data processing. AI techniques are known to exhibit higher accuracy as the amount of data increases. Depending on the characteristics of the data and the purpose of the analysis, small data processing may provide the required data analysis results.

\subsection{Hybrid Manufacturing Process}

For SMEs with limited-cost production facilities, it is necessary to introduce a process that can efficiently produce high-quality products with an appropriate cost expenditure. The hybrid process, which combines various production processes, is one of the most efficient ways to improve the performance and cost-effectiveness of the production process [42]. The potential benefits of the hybrid process are a reduced energy consumption, increased machining performance, and an increased machining accuracy [43]. To exploit these advantages, various hybrid processes have been developed and applied in the field [44, 45].

One of the most traditional methods for polishing materials is rubbing them with abrasive particles and polishing pads [46]. Because of the nature of polishing processing with a very low material removal rate (MRR), the processing time is inevitably long; thus, if the productivity and efficiency of the manufacturing process can be improved, the cost can be reduced.

Figure 6 compares the per-part manufacturing costs (electric power usage) and MRRs of various hybrid polishing processes using laser and ultrasonic vibration. Accordingly, the productivity and efficiency of the hybrid processes were compared. As shown, hybrid processes have higher productivity (MRR) and lower production costs than traditional processes, allowing more products to be produced simultaneously at lower costs through process hybridisation. 


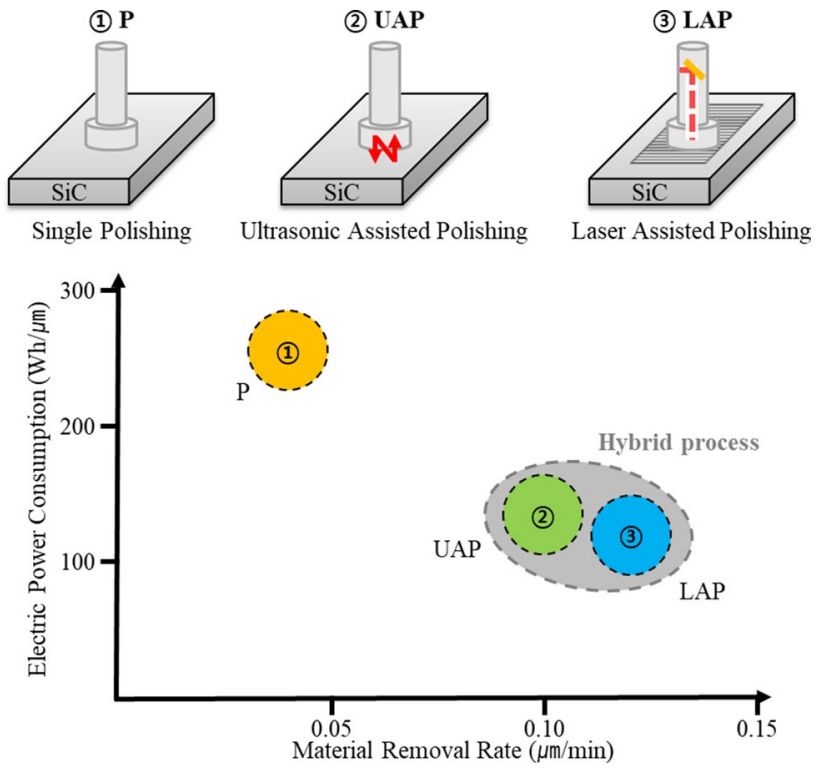

Fig. 6 Costs and MRRs for different polishing processes

\subsection{Appropriate Vision-Based Monitoring}

The range of applications of computer-vision technology has expanded rapidly as the resolution and frame rate of camera technology have increased. Additionally, image data processing technology utilising AI has been developed. However, the high cost of high-performance cameras and data-processing algorithms is a significant limiting factor for SMEs. In contrast, equipment that is $>20$ years old, which is employed by most SMEs, is often equipped with a builtin sensor that measures manufacturing data but often does not have a communication function that transmits the data to external databases. In such cases, manufacturing data are collected by installing incidentally middleware module-type data communication devices, which not only require equipment modification but also incur costs.

Figure 7 shows an example of the application of a lowcost webcam and open source-based image-processing technology with consideration of the situations faced by SMEs. The proper vision monitoring system was developed to digitise manufacturing data displayed on monitors of older machine tools and transmit them to the database. Thus, the equipment status can be monitored through vision technology without installing separate communication modules [47]. To ensure that the SMEs can accept the technology without a significant financial burden, low-cost webcams and small computers are used to monitor the status of older machine tools from mobile devices such as smartphones and tablets.
Fig. 7 Appropriate vision monitoring system for old machine tools
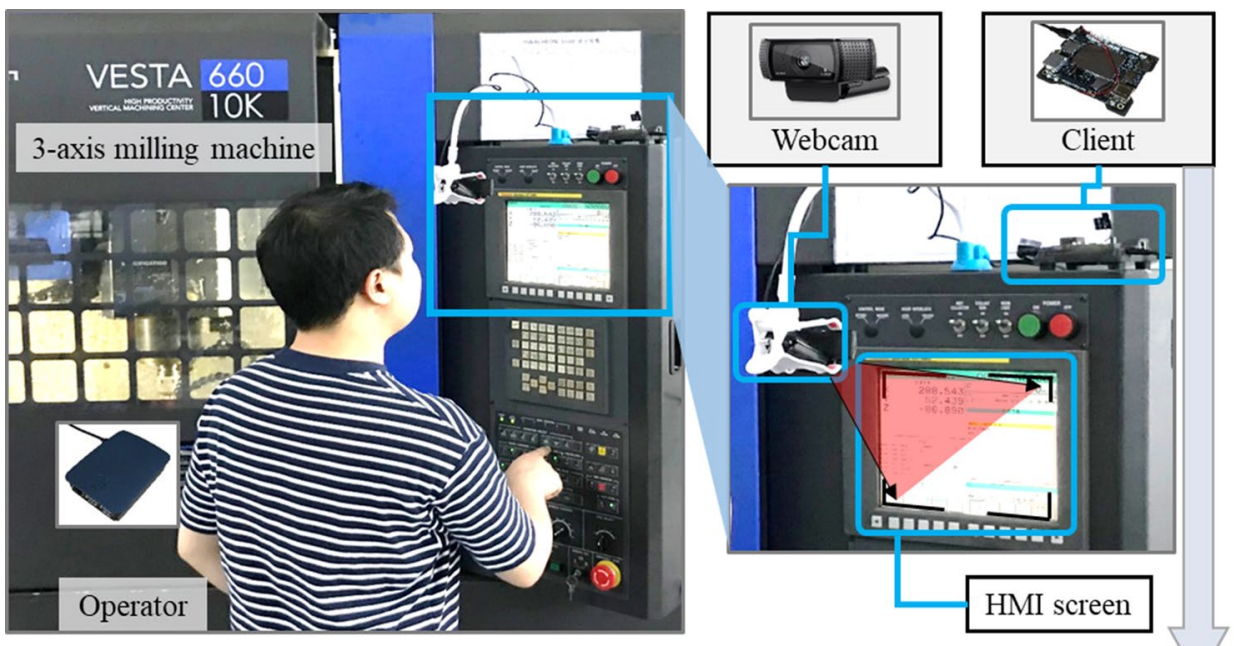

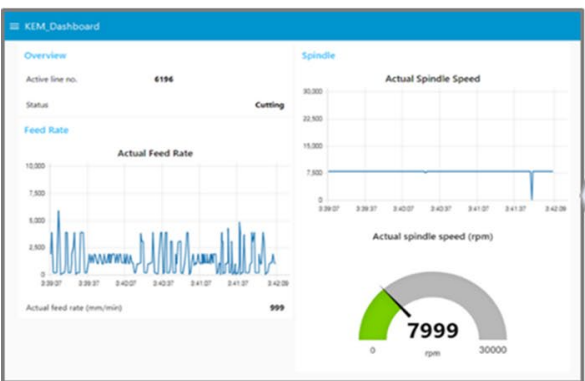

On-line dashboard

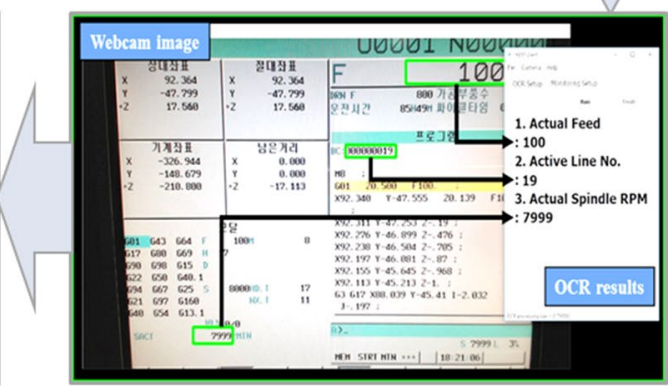

OCR process 


\section{Application of Appropriate Smart Factory Technologies}

Section 3 reviews the technical implementation methods for appropriate smart factories. Appropriate smart factory technologies that reflect EASI elements can be applied independently as a single technology but they can be more useful when applied in combination with their technical characteristics. This section presents a case study where a system is developed with consideration of the situations of SMEs by applying several appropriate smart factory technologies.

If a sewing-machine defect occurs in the middle of a garment sewing process, the defect remains until the final inspection stage. Defective products identified in quality inspection are sent back to the process after disassembly, incurring costs and resulting in time and manpower losses. A small and medium-sized sewing company attempted to analyse images of the sewing machine using AI to solve this problem and concluded that one expensive computer with a graphics processing unit (GPU) was needed for every five sewing machines. The analysis results for the SME requirements are presented in Table 3. In this section, the aforementioned problem is solved by combining IoT and computer vision, as well as small-volume data processing with the appropriate smart factory concept.

\subsection{Hardware Configuration}

Regarding the requirements in Table 3, the composition of the hardware requires appropriate vision monitoring with low-cost small cameras and small computers to handle the appropriate IoT and the small amount of data. Among the various commercially available models, the Raspberry Pi was selected as the small computer because it is relatively inexpensive and can satisfy the requirements.

Figure 8 shows a schematic of the hardware system configuration and the inspection device to be developed accordingly. The work conducted on the sewing machine was filmed using a small camera. Then, after image processing

Table 3 SME's requirements for the sewing quality inspection system

\begin{tabular}{ll}
\hline Category & Requirements \\
\hline Hardware & Price of $<300$ USD \\
& Easy to use and not \\
& affected by the machine \\
& model \\
Software & Configurable computing \\
& source within the price \\
& range \\
& Accuracy of $\geq 90 \%$ \\
& Defect judgment within $3 \mathrm{~s}$ \\
\hline
\end{tabular}

and fault detection on a small computer, an alarm was sounded by the appropriate IoT in the case of a defect, and the same operation was repeated if normal (Fig. 8a). Figure $8 \mathrm{~b}$ shows the result of configuring the system using the Raspberry Pi and Pi camera.

\subsection{Software Configuration}

The sewing inspection algorithm was designed to be run at a small-data level using a small computer. Algorithms were established for processing and judging image data collected from the hardware using OpenCV and Python, which are open-source solutions for image processing.

Figure 9 presents a flowchart of the data-processing algorithm. Captured images were treated with the canny edge detection method to extract the stitching characteristics and compare them with the characteristics of normal operations for detecting differences. If the stitching characteristics are deemed normal, the process continues until the sewing operation is complete, and if a fault occurs beyond the threshold, the system sends a 'stop' signal. Canny edge detection is a typical solution provided with the OpenCV application programming interface.

\subsection{Results}

The developed system was used to perform canny edge processing by taking four pictures per second according to the speed of the sewing machine, and the results are shown in Fig. 10. Noise from pictures captured by the hardware was eliminated first (Fig. 10a). Then, a simplified contour image was obtained through the processing of small amounts of data (Fig. 10b).

Figure 11 shows the result of the shape similarity evaluation of the processed image. The parameter study indicated that a shape distance of $\geq 2$ corresponded to a defect, and 2 of the total 20 images were found to have a shape distance of $\geq 2$. Both images were poor with regard to the image-processing results, but in one of them, the stitching was blurred owing to the rapid operation of the sewing machine. In our dataset, there was a case where an edge was not produced owing to an image shooting problem, which was not detected as a defect. Overall, two hardware problems and one software problem were identified for the 20 images, corresponding to $90 \%$ hardware reliability and $95 \%$ software reliability.

Table 4 compares the time spent for the small data processing methods used in this study for holistically nested edge detection (HED), which is an AI-based image-processing technique. The small data processing took a total of $1.23 \mathrm{~s}$ from image acquisition to similarity measurement and was adequate for determining the fault within $3 \mathrm{~s}$. However, in the case of HED, it took a total of $3.16 \mathrm{~s}$, slightly exceeding the limit. 
Fig. 8 Vision-based sewing quality inspection device. a Schematic of the hardware configuration, b configured sewing quality inspection device hardware

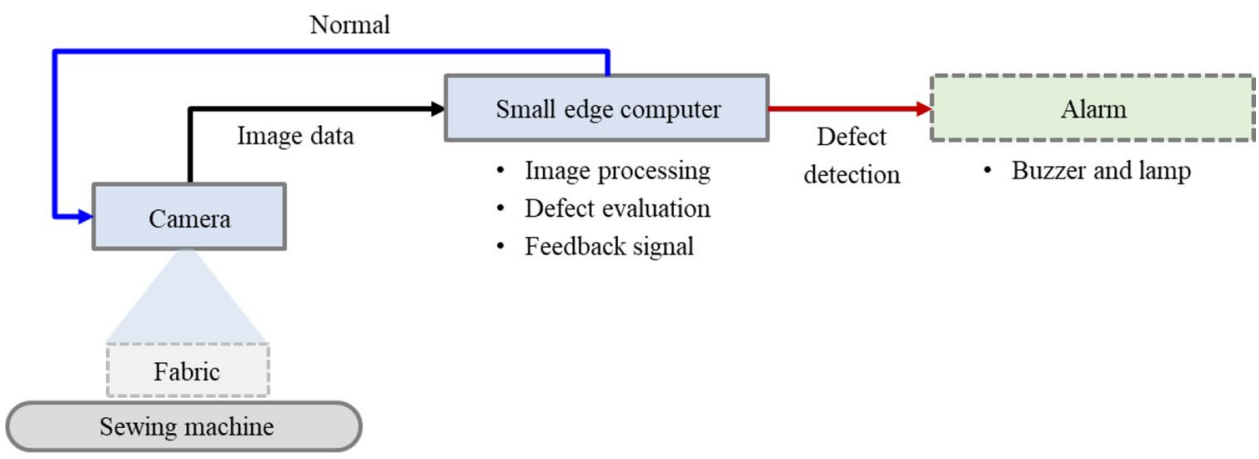

(a) Schematic of the hardware configuration

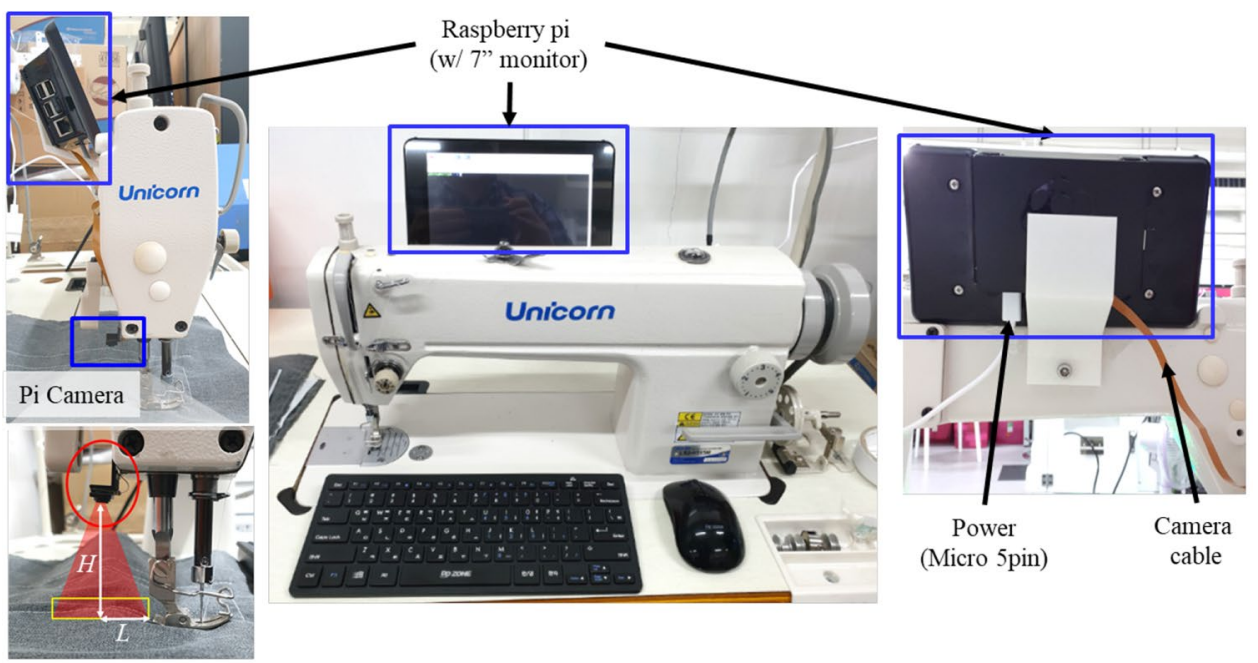

(b) Configured sewing quality inspection device hardware

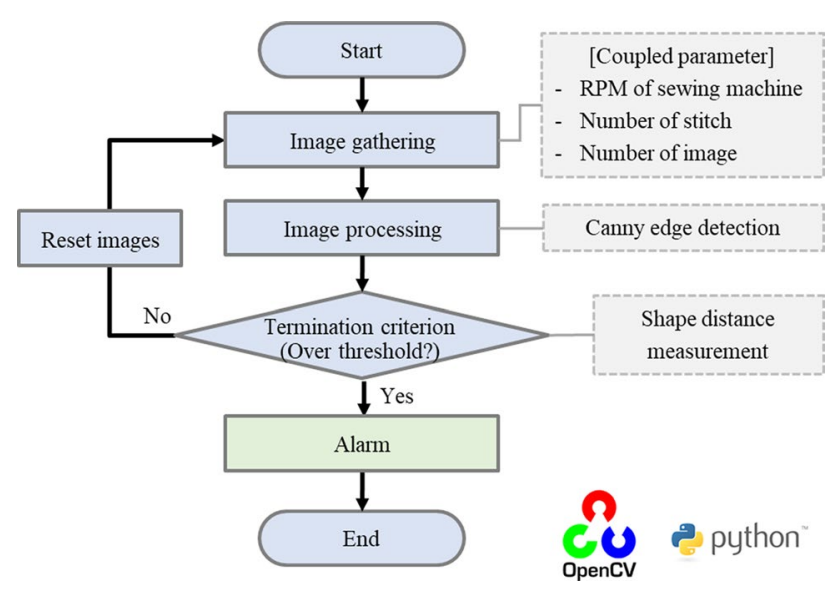

Fig. 9 Sewing quality inspection algorithm flow chart

\subsection{Requirements Satisfaction Assessment}

The requirements for the developed suture inspection device were evaluated using the proposed EASI approach. For the hardware, an affordable, essential, and simple solution was required. The complete system, including a computing unit and cameras, can be configured for $\leq 170$ USD. Various sewing tasks were performed using the developed system, and the system was operated easily without interruption.

For the smart data evaluation, a recognition rate of $>90 \%$ and a maximum response delay of $3 \mathrm{~s}$ until a fault determination was published were required. These requirements were satisfied, with an average recognition rate of $92.5 \%$ (recognition rate of hardware: $90 \%$; recognition rate of software: 95\%). The requirement for fault determination within $3 \mathrm{~s}$ was satisfied by the small data processing method (1.23 s) (Simple), as previously mentioned, but the AI-based algorithms could not satisfy the requirements in our case study, given the tooling and development time provided. While AI-based methods may be more suitable for the analysis of complex shapes, small data processing methods based on numerical computing can be more useful for simple image analysis using low-cost consumer-based computing units.

The sewing quality inspection system developed in this study aims to increase productivity while improving the 
Fig. 10 Sewing image acquisition and processing results. a Photographs captured at the sewing-machine speed (after noise removal), b photographs after the basic image processing

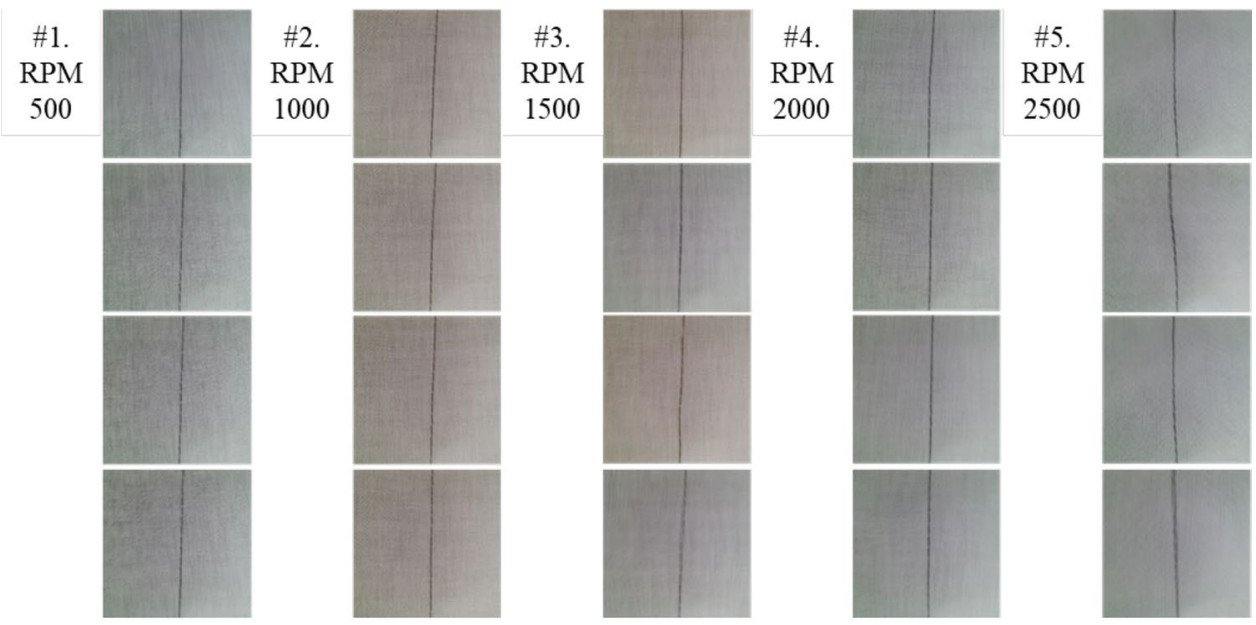

(a) Photographs captured at the sewing-machine speed (after noise removal)

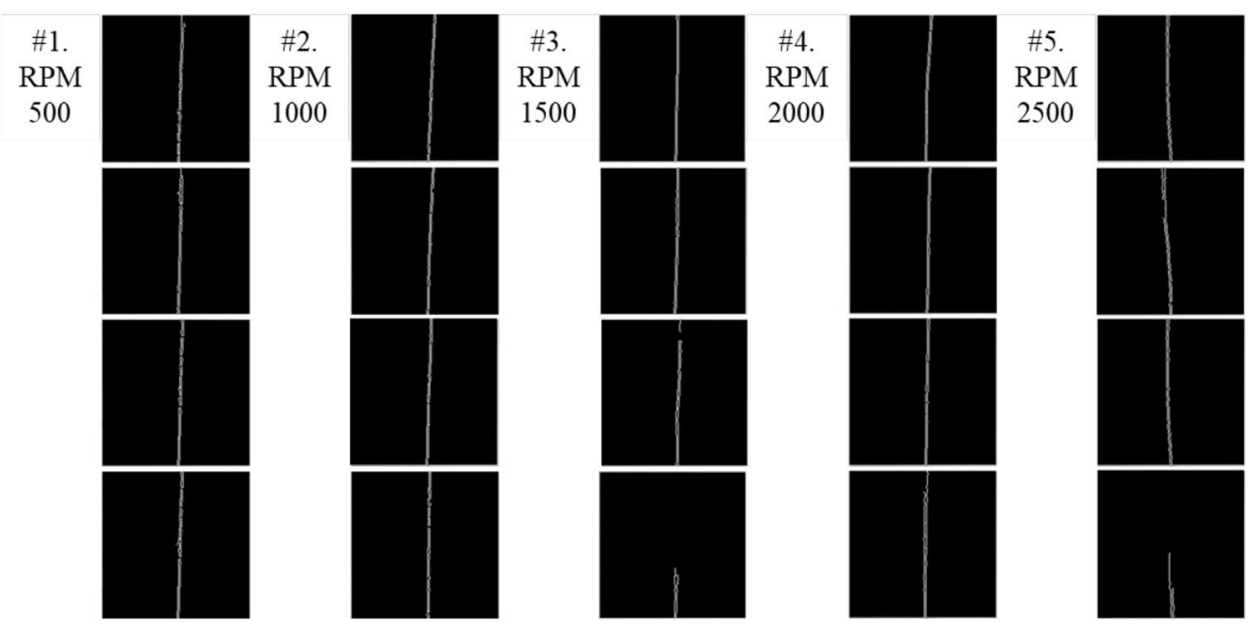

(b) Photographs after the basic image processing

Fig. 11 Similarity distance measurement results

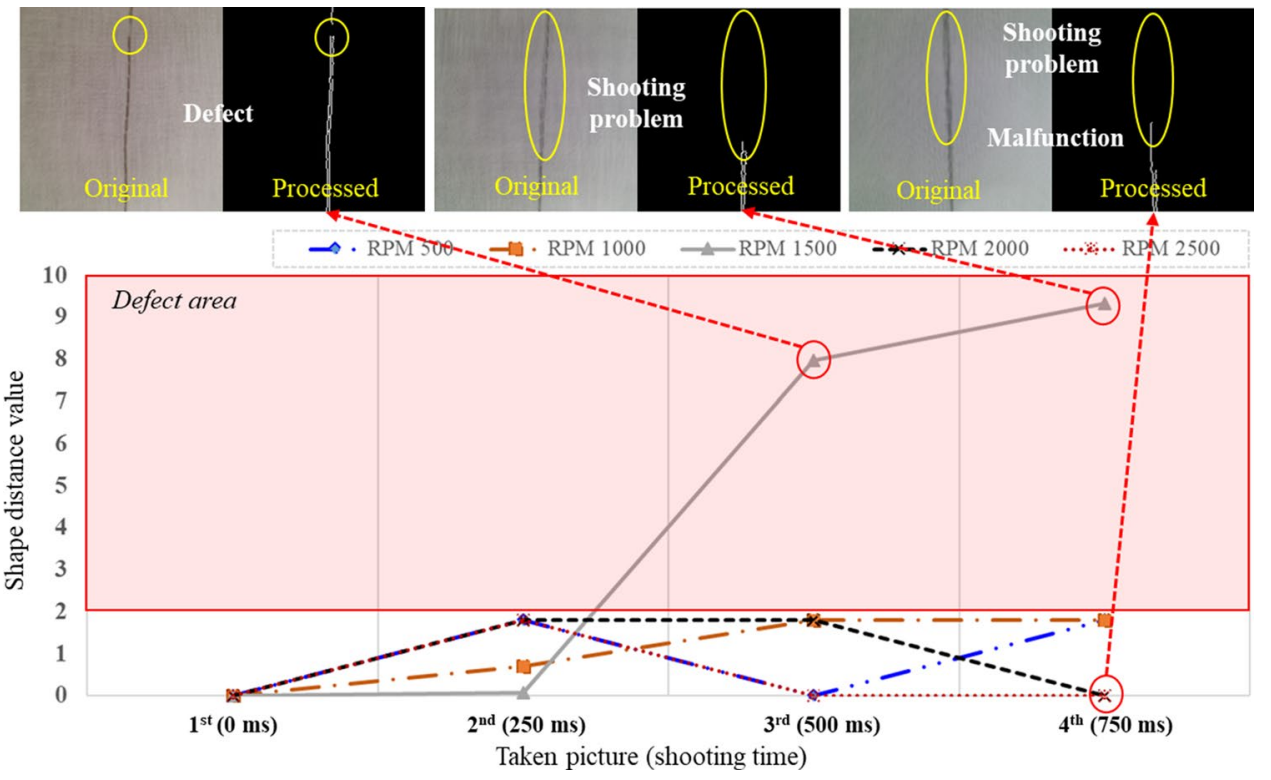


Table 4 Comparison of sewing image processing time

\begin{tabular}{lll}
\hline Process & $\begin{array}{l}\text { Small data processing (OpenCV } \\
\text { canny edge) }\end{array}$ & $\begin{array}{l}\text { AI-based processing } \\
\text { (holistically nested edge } \\
\text { detection) }\end{array}$ \\
\hline Image gathering & $1000 \mathrm{~ms}$ & Avg. $2060 \mathrm{~ms}$ \\
Image processing & Avg. $130 \mathrm{~ms}$ & $3160 \mathrm{~ms}$ \\
Similarity measurement & Avg. $100 \mathrm{~ms}$ & $1230 \mathrm{~ms}$ \\
Total & &
\end{tabular}

sewing quality of garment. The garment production system consists of production lines in a pipeline structure. Figure 12 shows an example of a typical garment production line. If a sewing defect occurs in the middle of such production line, it is confirmed in the final inspection and a lot of time and effort is wasted in the process of moving back to the original process and correcting it. Assuming that the example line is a line that produces about 250 hooded jackets per day, when the time information of the sewing line is considered [48], sewing defects cause an average production time delay of 30.75 min per product. Applying 5\%, which is the incidence of sewing defects in a typical garment production line, the example line can save an average of 384 min per day of the total available time. In addition to the time savings, of course, the level of sewing quality will be also improved by the monitoring.

In summary, we examined a case study for the development of a sewing product inspection device that combines IoT, vision, and data processing on low-cost consumer hardware. While further studies on the sewing environment should be performed to improve the system, the results indicated that systems that conform to the appropriate smart factory concept can satisfy the requirements of SMEs.

\section{Discussion: Perspective of Appropriate Smart Factory}

Thus far, we have considered the concept of appropriate smart factories and examples of technology implementation that allow SMEs to introduce smart factories for avoiding financial and technical obstacles. However, smart factories cannot be introduced to SMEs simply by introducing new technologies. Few SMEs have the ability to ignore the current system and introduce new systems.

Table 5 presents four important factors in evaluating smart-factory elements suitable for investments in equipment that is currently used by SMEs. With the introduction of suitable retrofit solutions, companies should be able to leverage the benefits of smart factories more efficiently without excessive financial investment.

Safety is also an important issue for smart factories. Many industrial accidents occur at manufacturing sites when the safety locks of equipment are manipulated to improve productivity or achieve work convenience, as well as when safety instructions are ignored completely. Appropriate smart solutions can be used to enhance the safety of these factories. Appropriate vision monitoring and computing modules can be used to detect hazardous situations and provide warnings, as well as monitor and highlight older equipment in cases where safety locks may be manipulated. When appropriate smart factory technology developers and field managers develop ideas tailored to the situations and facilities at the site, a safer environment can be realised. However, as learning-enabled components are introduced to manufacturing processes, it is challenging to ensure absolute safety in decision-making for smart factory operation. This issue is becoming more important when fully automating factories. Modern decision-making technologies can contribute to resolving safety issues in automated manufacturing processes. These include tools for safety specification $[49,50]$, risk-aware optimization [51, 52], and safe learning [53]. In particular, a recent distributionally robust optimization technology may enhance
Fig. 12 Example of the flow of sewing defects in the garment production line

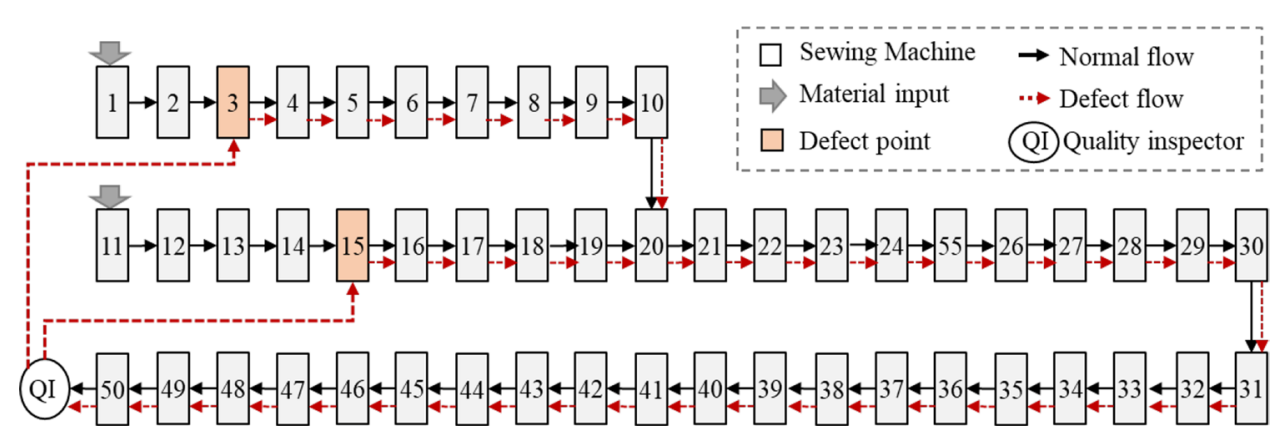


Table 5 Appropriate smart factory guidelines as important considerations for SMEs

\begin{tabular}{|c|c|}
\hline Factors & Problems and alternatives \\
\hline Essential & $\begin{array}{l}\text { Unused functions: minimise maintenance by removing or simplifying unused functions } \\
\text { Transient specification facilities: adopt essential functional specifications when replacing or introducing new facilities }\end{array}$ \\
\hline Affordable & $\begin{array}{l}\text { Poor cost-effectiveness: integrate review with equipment compatibility items to determine whether it can be used together in other } \\
\text { production lines } \\
\text { Limited current financial acceptance of facility replacement and new adoption: review mid- to long-term expected operational } \\
\text { maintenance costs and expected profits, and determination of reduced operation of facilities and systems }\end{array}$ \\
\hline Simple & $\begin{array}{l}\text { Restrictions on maintenance and difficulties in operation: intensive training on essential functions and checks for operators and } \\
\text { maintenance engineers } \\
\text { Excess installation of incidental facilities/systems: consideration of ease of installation, maintenance, and operation in designing } \\
\text { and adopting facilities/systems }\end{array}$ \\
\hline Interoperable & $\begin{array}{l}\text { Facility/system and communication module interoperability restrictions: development/application of standardised compatibility } \\
\text { modules with minimum-cost incidental devices } \\
\text { Part/configuration module interoperability limits: consideration of standardisation in designing/upgrading lines } \\
\text { System-to-system compatibility restrictions due to disparate data types: defining and utilising standard forms for all the opera- } \\
\text { tional data of the factory }\end{array}$ \\
\hline
\end{tabular}

safety in operation of autonomous systems when using inaccurate learning results [54].

Another issue concerns smart-factory technology development and supply companies. If supply companies develop smart-factory technology in the conceptual design phase, taking into account the financial, technical, and field conditions of SMEs at the appropriate smart factory level, the companies that purchase and use this technology will be greatly satisfied, resulting in a mutual benefit in the manufacturing ecosystem.

\section{Conclusions}

Case studies involving technology applications were examined in conjunction with our 'appropriate smart factory' concept, which includes essential functions but is an economically acceptable, easy to use, and compatible method of implementing smart factories for SMEs. Appropriate smart factories should apply the 'EASI' method to minimise financial and technical burdens that SMEs face in adopting smart factories. The proposed method is also useful for implementing customised technology on manufacturing sites by combining sensors, IoT, process mixing, and data processing.

In the manufacturing industry, smart factories embody the final stage of the Fourth Industrial Revolution. In the rapidly changing global environment, small and medium-sized manufacturing companies cannot secure global competitiveness with the same strategies as large enterprises. Under these circumstances, the appropriate smart factory concept can be used by SMEs to remain competitive. Applied research at local universities and fruitful international cooperation with leading institutes can reduce development costs, showcase industrial solutions for orientation and adoption, and foster global and open standards for improved compatibility, which would significantly benefit SMEs. We hope that the introduction of appropriate smart factories will allow SMEs to overcome the technical and financial obstacles that they currently face.

Acknowledgements This work was supported by the Basic Research Lab Program through the National Research Foundation of Korea (NRF) funded by the Ministry of Science and ICT (MSIT) (2018R1A4A1059976); Korea Basic Science Institute (KBSI) Creative Convergence Research Project (CAP15-01-KBSI) funded by the National Research Council of Science and Technology (NST); and the Safe Smart Factory Demonstration Program funded by Gyeonggi Province (AICT-2020-0057); and the SNU-Hojeon Garment Smart Factory Research Center funded by the Hojeon Ltd. (SNU-0423-20190068); and the Basic Science Research Program through the National Research Foundation of Korea (NRF) funded by the Ministry of Education (2020R1A6A3A01099046).

Open Access This article is licensed under a Creative Commons Attribution 4.0 International License, which permits use, sharing, adaptation, distribution and reproduction in any medium or format, as long as you give appropriate credit to the original author(s) and the source, provide a link to the Creative Commons licence, and indicate if changes were made. The images or other third party material in this article are included in the article's Creative Commons licence, unless indicated otherwise in a credit line to the material. If material is not included in the article's Creative Commons licence and your intended use is not permitted by statutory regulation or exceeds the permitted use, you will need to obtain permission directly from the copyright holder. To view a copy of this licence, visit http://creativecommons.org/licenses/by/4.0/.

\section{References}

1. Wittenberg, C. (2016). Human-CPS interaction-requirements and human-machine interaction methods for the Industry 4.0. IFACPapersOnLine, 49(19), 420-425.

2. Monostori, L., Kádár, B., Bauernhansl, T., Kondoh, S., Kumara, S., Reinhart, G., et al. (2016). Cyber-physical systems in manufacturing. CIRP Annals, 65(2), 621-641. 
3. Büttner, K.-H., \& Brück, U. (2017). Use Case Industrie 4.0-Fertigung im Siemens Elektronikwerk Amberg, Handbuch Industrie 4.0 Bd.4, pp. 45-70. https://doi.org/10.1007/978-3-662-53254 $-6 \_3$.

4. Lu, Y., \& Xu, X. (2018). Resource virtualization: A core technology for developing cyber-physical production systems. Journal of Manufacturing Systems, 47, 128-140.

5. Francalanza, E., Borg, J., \& Constantinescu, C. (2017). A knowledge-based tool for designing cyber physical production systems. Computers in Industry, 84, 39-58.

6. Lee, J., Jin, C., \& Bagheri, B. (2017). Cyber physical systems for predictive production systems. Production Engineering, 11(2), 155-165.

7. Elduque, A., Elduque, D., Clavería, I., \& Javierre, C. (2018). Influence of Material and Injection Molding Machine's selection on the electricity consumption and environmental impact of the injection molding process: An experimental approach. International Journal of Precision Engineering and Manufacturing-Green Technology, 5(1), 13-28.

8. Nam, J., \& Lee, S. W. (2018). Machinability of titanium alloy (Ti-6Al-4 V) in environmentally-friendly micro-drilling process with nanofluid minimum quantity lubrication using nanodiamond particles. International Journal of Precision Engineering and Manufacturing-Green Technology, 5(1), 29-35.

9. Peng, T., \& Chen, C. (2018). Influence of energy density on energy demand and porosity of 316L stainless steel fabricated by selective laser melting. International Journal of Precision Engineering and Manufacturing-Green Technology, 5(1), 55-62.

10. Jackson, M. A., Van Asten, A., Morrow, J. D., Min, S., \& Pfefferkorn, F. E. (2018). Energy consumption model for additive-subtractive manufacturing processes with case study. International Journal of Precision Engineering and Manufacturing-Green Technology, 5(4), 459-466.

11. Wang, Q., Tang, D., Yin, L., Ullah, I., Tan, L., \& Zhang, T. (2018). An optimization model for low carbon oriented modular product platform planning (MP 3). International Journal of Precision Engineering and Manufacturing-Green Technology, 5(1), 121-132.

12. Hong, M.-P., Kim, W.-S., Sung, J.-H., Kim, D.-H., Bae, K.-M., \& Kim, Y.-S. (2018). High-performance eco-friendly trimming die manufacturing using heterogeneous material additive manufacturing technologies. International Journal of Precision Engineering and Manufacturing-Green Technology, 5(1), 133-142.

13. Chaitanya, S., \& Singh, I. (2018). Ecofriendly treatment of aloe vera fibers for PLA based green composites. International Journal of Precision Engineering and Manufacturing-Green Technology, 5(1), 143-150.

14. Joe, H.-E., Yun, H., Jo, S.-H., Jun, M. B., \& Min, B.-K. (2018). A review on optical fiber sensors for environmental monitoring. International Journal of Precision Engineering and Manufacturing-Green Technology, 5(1), 173-191.

15. Ministry of SMEs and Startups. (2020). Korean SME statistics (2020). Retrieved October 21, 2020, from https://www.mss.go.kr/ site/smba/foffice/ex/statDB/MainSubStat.do/.

16. Jung, E. M. (2018). Influences and Major Tasks of the 4th Industrial Revolution on Major Industries. Industrial Economics Analysis, Korea Institute for Industrial Economics \& Trade, 44-57.

17. Ok, K. J. (2020). Interview with Vice President of Taelim Industry. July 13, 2020.

18. Mittal, S., Khan, M. A., Purohit, J. K., Menon, K., Romero, D., \& Wuest, T. (2020). A smart manufacturing adoption framework for SMEs. International Journal of Production Research, 58(5), $1555-1573$.

19. Zuehlke, D. (2010). SmartFactory-Towards a factory-of-things. Annual Reviews in Control, 34(1), 129-138.
20. Wang, S., Wan, J., Li, D., \& Zhang, C. (2016). Implementing smart factory of industrie 4.0: An outlook. International Journal of Distributed Sensor Networks, 12(1), 3159805.

21. Chen, B., Wan, J., Shu, L., Li, P., Mukherjee, M., \& Yin, B. (2017). Smart factory of industry 4.0: Key technologies, application case, and challenges. IEEE Access, 6, 6505-6519.

22. Kang, H. S., Lee, J. Y., Choi, S., Kim, H., Park, J. H., Son, J. Y., et al. (2016). Smart manufacturing: Past research, present findings, and future directions. International Journal of Precision Engineering and Manufacturing-Green Technology, 3(1), 111-128.

23. Sim, H. S. (2019). Big data analysis methodology for smart manufacturing systems. International Journal of Precision Engineering and Manufacturing, 20(6), 973-982.

24. Kim, D.-H., Kim, T. J., Wang, X., Kim, M., Quan, Y.-J., Oh, J. W., et al. (2018). Smart machining process using machine learning: A review and perspective on machining industry. International Journal of Precision Engineering and Manufacturing-Green Technology, 5(4), 555-568.

25. Shin, I., Lee, J., Lee, J. Y., Jung, K., Kwon, D., Youn, B. D., et al. (2018). A framework for prognostics and health management applications toward smart manufacturing systems. International Journal of Precision Engineering and Manufacturing-Green Technology, 5(4), 535-554.

26. Kim, J. S., Lee, C. S., Kim, S.-M., \& Lee, S. W. (2018). Development of data-driven in situ monitoring and diagnosis system of fused deposition modeling (FDM) process based on support vector machine algorithm. International Journal of Precision Engineering and Manufacturing-Green Technology, 5(4), 479-486.

27. Pham, A.-D., \& Ahn, H.-J. (2018). High precision reducers for industrial robots driving 4th industrial revolution: state of arts, analysis, design, performance evaluation and perspective. International Journal of Precision Engineering and ManufacturingGreen Technology, 5(4), 519-533.

28. Grube, D., Malik, A. A., \& Bilberg, A. (2017). Generic Challenges and Automation Solutions in Manufacturing SMEs. In B. Katalinic (Ed.), Proceedings of the 28th DAAAM International Symposium (pp. 1161-1169). Vienna, Austria: DAAAM International. ISBN 978-3-902734-11-2, ISSN 1726-9679. https://doi. org/10.2507/28th.daaam.proceedings.161.

29. Rauch, E., Dallasega, P., \& Unterhofer, M. (2019). Requirements and barriers for introducing smart manufacturing in small and medium-sized enterprises. IEEE Engineering Management Review, 47(3), 87-94.

30. Tao, F., Qi, Q., Liu, A., \& Kusiak, A. (2018). Data-driven smart manufacturing. Journal of Manufacturing Systems, 48, 157-169.

31. Mittal, S., Khan, M. A., Romero, D., \& Wuest, T. (2018). A critical review of smart manufacturing \& Industry 4.0 maturity models: Implications for small and medium-sized enterprises (SMEs). Journal of Manufacturing Systems, 49, 194-214.

32. Issa, A., Lucke, D., \& Bauernhansl, T. (2017). Mobilizing SMEs towards Industrie 4.0-enabled smart products. Procedia CIRP, 63, 670-674.

33. Chonsawat, N., \& Sopadang, A. (2019). The development of the maturity model to evaluate the smart SMEs 4.0 readiness. In Proceedings of the international conference on industrial engineering and operations management (pp. 5-7). Bangkok, Thailand: JW Marriott Hotel Bangkok.

34. Ghobakhloo, M., \& Ching, N. T. (2019). Adoption of digital technologies of smart manufacturing in SMEs. Journal of Industrial Information Integration, 16, 100107.

35. Jun, C., Lee, J. Y., Yoon, J.-S., \& Kim, B. H. (2017). Applications' integration and operation platform to support smart manufacturing by small and medium-sized enterprises. Procedia Manufacturing, 11, 1950-1957. 
36. Menezes, S., Creado, S., \& Zhong, R. Y. (2018). Smart manufacturing execution systems for small and medium-sized enterprises. Procedia CIRP, 72, 1009-1014.

37. Kolla, S., Minufekr, M., \& Plapper, P. (2019). Deriving essential components of lean and industry 4.0 assessment model for manufacturing SMEs. Procedia CIRP, 81, 753-758.

38. Ahn, S.-H. (2018). Retrieved October 21, 2020, from https://www. hankyung.com/opinion/article/2018092111451.

39. Min, S.-H., Kim, H.-J., Quan, Y.-J., Kim, H.-S., Lyu, J.-H., Lee, G.-Y., et al. (2020). Stretchable chipless RFID multi-strain sensors using direct printing of aerosolised nanocomposite. Sensors and Actuators, A: Physical, 313, 112224.

40. Min, S.-H., Lee, G.-Y., \& Ahn, S.-H. (2019). Direct printing of highly sensitive, stretchable, and durable strain sensor based on silver nanoparticles/multi-walled carbon nanotubes composites. Composites Part B Engineering, 161, 395-401.

41. Jung, W.-K., Kim, H. K., Park, Y.-C., Lee, J.-W. L., \& Ahn, S.-H. (2019). Smart sewing work measurement system using IoT-based power monitoring device and approximation algorithm. International Journal of Production Research, 58, 15-44.

42. Serrano, P. A. A., Kim, M., Kim, D.-R., Kim, D.-H., Kim, G.-H., \& Ahn, S.-H. (2020). Spherical mirror and surface patterning on silicon carbide $(\mathrm{SiC})$ by material removal rate enhancement using $\mathrm{CO}_{2}$ laser assisted polishing. International Journal of Precision Engineering and Manufacturing, 21, 775-785.

43. Chu, W.-S., Kim, M.-S., Jang, K.-H., Song, J.-H., Rodrigue, H., Chun, D.-M., et al. (2016). From design for manufacturing (DFM) to manufacturing for design (MFD) via hybrid manufacturing and smart factory: A review and perspective of paradigm shift. International Journal of Precision Engineering and ManufacturingGreen Technology, 3, 209-222.

44. Yong-Hun, J., Ahn, D.-G., Kim, J. K., \& Kim, W.-S. (2018). Remelting characteristics of a stellite 21 deposited part by direct energy deposition process using a pulsed plasma electron beam with a large irradiation area. International Journal of Precision Engineering and Manufacturing-Green Technology, 5, 467-477.

45. Oh, N.-S., Woo, W.-S., \& Lee, C.-M. (2018). A study on the machining characteristics and energy efficiency of Ti-6Al-4 V in laser-assisted trochoidal milling. International Journal of Precision Engineering and Manufacturing-Green Technology, 5, $37-45$.

46. Kim, M., Bang, S., Kim, D.-H., Lee, H.-T., Kim, G.-H., \& Ahn, S.-H. (2020). Hybrid $\mathrm{CO}_{2}$ laser-polishing process for improving material removal of silicon carbide. The International Journal of Advanced Manufacturing Technology, 106, 3139-3151.

47. Kim, H., Jung, W.-K., Choi, I.-G., \& Ahn, S.-H. (2019). A lowcost vision-based monitoring of computer numerical control (CNC) machine tools for small and medium-sized enterprises (SMEs). Sensors, 19, 1-18.

48. Jung, W.-K., Kim, H., Park, Y.-C., Lee, J.-W., \& Suh, E. S. (2020). Real-time data-driven discrete-event simulation for garment production lines. Production Planning \& Control. https://doi. org/10.1080/09537287.2020.1830194.

49. Lygeros, J., Tomlin, C., \& Sastry, S. (1999). Controllers for reachability specifications for hybrid systems. Automatica, 35(3), 349-370.

50. Yang, I. (2018). A dynamic game approach to distributionally robust safety specifications for stochastic systems. Automatica, 94, 94-101.

51. Blackmore, L., Ono, M., \& Williams, B. C. (2011). Chance-constrained optimal path planning with obstacles. IEEE Transactions on Robotics, 27(6), 1080-1094.

52. Hakobyan, A., Kim, G. C., \& Yang, I. (2019). Risk-aware motion planning and control using CVaR-constrained optimization. IEEE Robotics and Automation Letters, 4(4), 3924-3931.
53. Hewing, L., Wabersich, K. P., Menner, M., \& Zeilinger, M. N. (2020). Learning-based model predictive control: Toward safe learning in control. Annual Review of Control, Robotics, and Autonomous Systems, 3, 269-296.

54. Hakobyan, A., \& Yang, I. (2020). Learning-based distributionally robust motion control with Gaussian processes. In Proceedings of the 2020 IEEE/RSJ international conference on intelligent robots and systems (IROS).

Publisher's Note Springer Nature remains neutral with regard to jurisdictional claims in published maps and institutional affiliations.

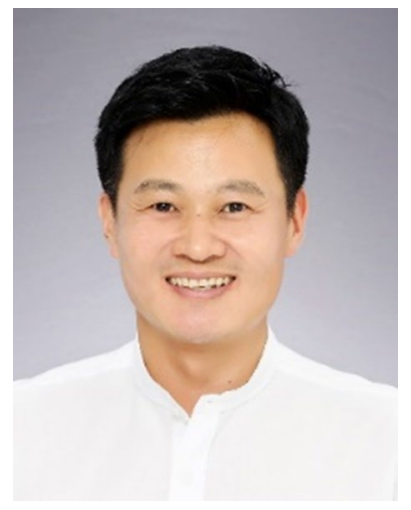

Woo-Kyun Jung received a Ph.D. in Mechanical Engineering from the Seoul National University in 2020. He was an army officer of R.O.K. from 1997 to 2017 and retired in 2017 as a lieutenant colonel. Prior to retirement to lieutenant colonel, he served as technical staff, military logistics school instructor, platoon leader, company commander, and battalion commander. Jung's research interests are in smart factory, soft robotics, smart/ composite materials, and approover 20 journal articles in these areas. priate technology. He published

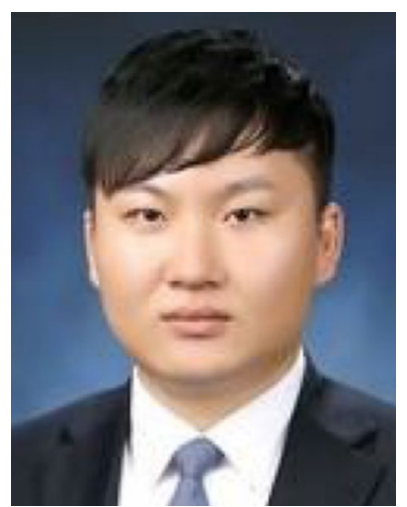

Dong-Ryul Kim Ph.D. candidate in the Department of Mechanical Engineering, Seoul National University. His main research interest is hybrid manufacturing process, such as Ultrasonic vibration assisted polishing and laser assisted polishing.

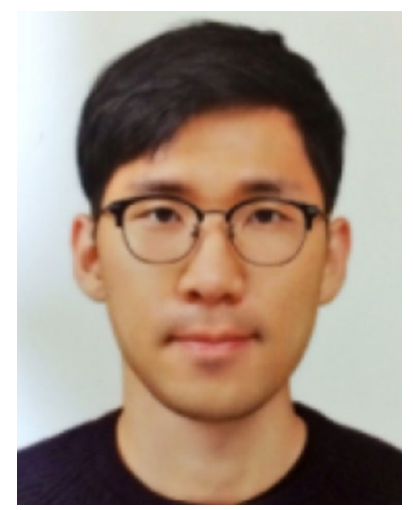

Hyunsu Lee received his B.S. degree and M.S degree from the School of Electrical and Electronics Engineering, Chung-Ang University, Seoul, Korea, in 2015 and 2018. He is currently $\mathrm{Ph} . \mathrm{D}$. candidate in the Department of Mechanical Engineering, Seoul National University. His main research interest are artificial intelligence, intelligent control and machine vision for s mart manufacturing technology. 


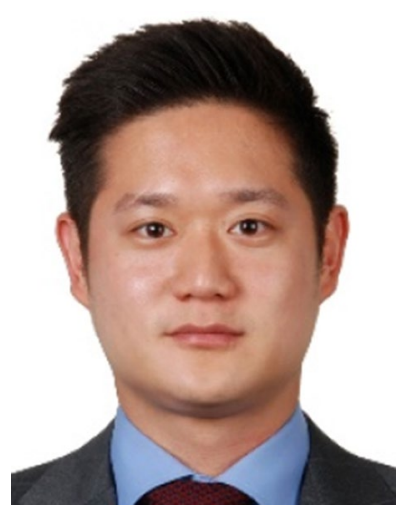

Tae-Hun Lee has been an employee of the Fraunhofer Institute for Production Technology IPT since 2015. He is responsible for "Machine characterization" and "Calibration of the machine tools" at the Fraunhofer IPT. Since 2018, he took up the position as Manager of the Machine Development and Machine Networking Group.

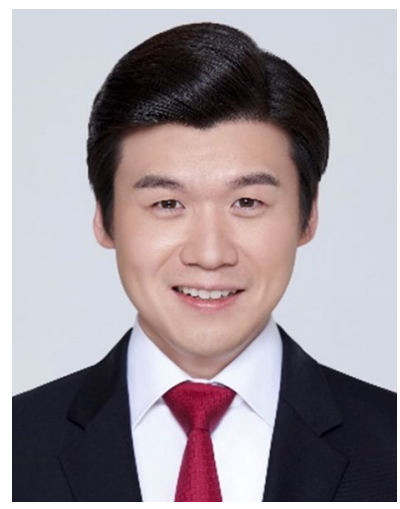

Insoon Yang is an Associate Professor in the Department of Electrical and Computer Engineering at Seoul National University (SNU). He received B.S. degrees in Mathematics and in Mechanical Engineering (summa cum laude) from SNU in 2009; and an M.S. in EECS, an M.A. in Mathematics and a Ph.D. in EECS from UC Berkeley in 2012, 2013 and 2015 , respectively. He was an Assistant Professor in the ECE department at USC from 2016 to 2018 and a Postdoctoral Associate with the Laboratory for Information and Decision Systems at MIT from 2015 to 2016. His research interests are in stochastic control and optimization, and reinforcement learning, with application to cyber-physical systems and safe autonomy. He is a recipient of the 2015 Eli Jury Award and a finalist for the Best Student Paper Award at the 55th IEEE Conference on Decision and Control 2016. He is an associate editor of the IEEE CSS Conference Editorial Board and a vice-chair of the IFAC Stochastic Systems Technical Committee.

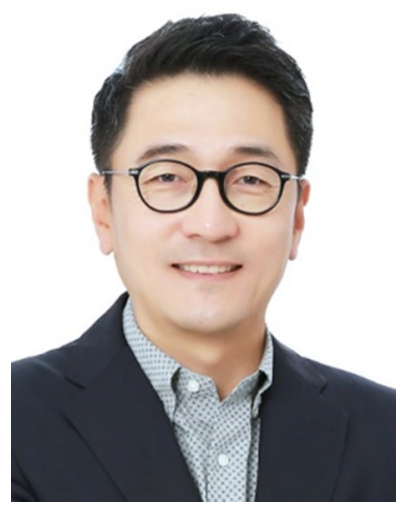

Byeng D. Youn received the Ph.D. degree from The University of Iowa in 2001 . He is currently a full Professor in the department of mechanical engineering at Seoul National University (SNU) in South Korea and the CEO of OnePredict Inc. He is a technical advisory board member of many global manufacturers including LG Electronics, Hyundai Motors, etc. He was affiliated with the University of Maryland, College Park, and the Michigan Technological University. His current research interests include prognostics and health management (PHM), reliability-based design, energy harvester design, and statistical model validation.

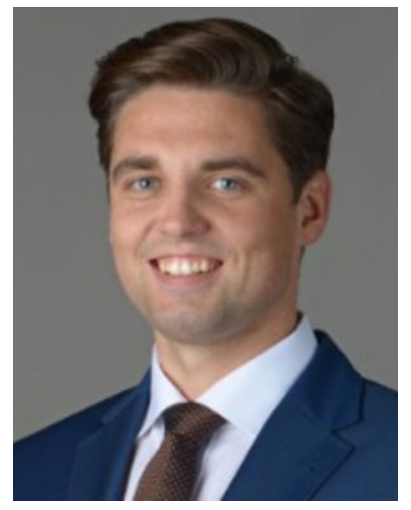

Daniel Zontar has been an employee of the Fraunhofer Institute for Production Technology IPT since 2009. In the period from 2014 to 2017 he was responsible for "Active alignment for the assembly of optical systems" at the Fraunhofer IPT and took up the position as Manager of the Precision Engineering and Automation Department in 2018. In his capacity as the Coordinator of International Research and Industry Projects, he has developed a range of technologies used to control assembly cells as well as solutions for special-purpose and classical machine tools.

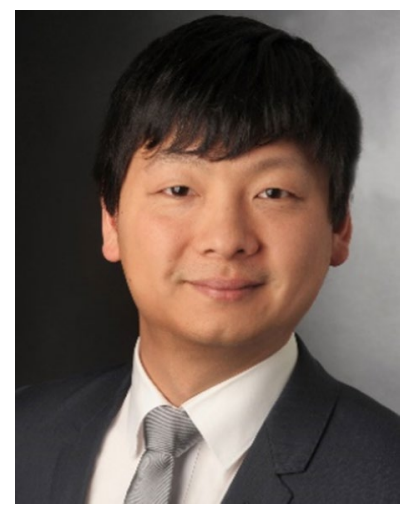

Dr. Matthias Brockmann is managing director of the Cluster of Excellence „Internet of Production" at RWTH Aachen University. Before this position, he was research group leader of "Product and Process Monitoring" group and Chief Engineer at the Chair of Manufacturing Technologies led by Prof. Fritz Klocke.

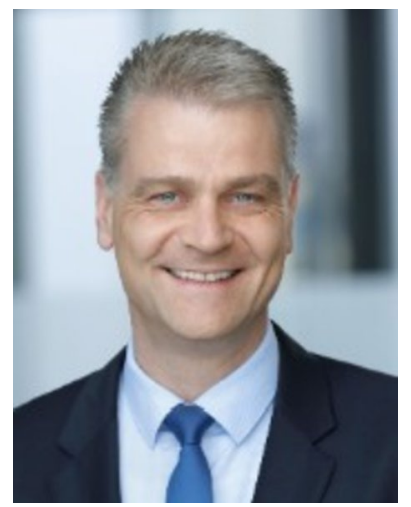

Christian Brecher On January, 2018, Prof. Dr.-Ing. Christian Brecher, has been appointed the Executive Director of the Fraunhofer Institute for Production Technology IPT. Since January, 2004, he has been the Ordinary Professor for Machine Tools at the Laboratory for Machine Tools and Production Engineering (WZL) of the RWTH Aachen as well as the Director of the Department for Production Machines at the Fraunhofer IPT.

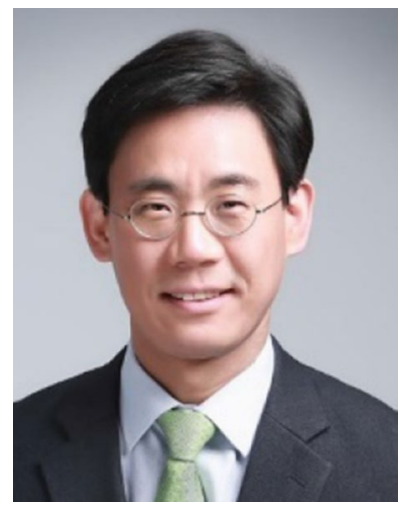

Sung-Hoon Ahn received a Ph.D. degree from Stanford University in 1997. He has been affiliated with UC Berkeley, Gyeongsang National University, University of Washington, and Hyundai WIA Corp. He is currently a full professor in the department of mechanical and aerospace engineering at Seoul National University. His research interests include smart factory, green manufacturing, soft robotics, 3D printing, composite materials, micro/nano fabrications, renewable energy, and appropriate technology. 